\title{
Vulnérabilité et stratégies endogènes de conservation des plantes utilisées dans le traitement du diabète dans les communes de Glazoué et Savè au Centre-Bénin
}

\author{
Iboukoun Fidèle LAWIN ${ }^{1 *}$, Obafemi Arnauld Fernand LALEYE $^{2}$ et \\ Onodjè Pierre AGBANI ${ }^{1}$ 526 Cotonou, Bénin.
${ }^{2}$ Laboratoire d'Enzymologie et de Biochimie des Protéines, Faculté des Sciences et Techniques, Université d'Abomey-Calavi, 05 BP 972 Cotonou, Bénin. \\ ${ }^{1}$ Laboratoire d'Ecologie Appliquée, Faculté des Sciences Agronomiques, Université d'Abomey-Calavi, 01 BP \\ *Auteur correspondant; E-mail : ifilawin@yahoo.fr; Tel.0022995114093/0022997181559
}

\section{RESUME}

Le prélèvement excessif des organes de plantes à des fins médicinales constitue un facteur de menace sur la phytodiversité. Cette étude réalisée dans les communes de Glazoué et Savè au Centre-Bénin, a pour objectif d'évaluer la vulnérabilité et les stratégies endogènes de conservation des plantes utilisées dans le traitement du diabète. A cet effet, des enquêtes ethnobotaniques ont été effectuées auprès de 126 personnes dont 35 femmes et 91 hommes. Les entretiens ont porté sur l'inventaire des plantes antidiabétiques; les différents organes utilisés et commercialisés et les stratégies endogènes de conservation desdites plantes. La méthode d'Evaluation Rapide de Vulnérabilité a été utilisée. Les résultats obtenus montrent que 144 plantes sont utilisées dans le traitement traditionnel du diabète. Trois niveaux de pression ont été distingués. Les niveaux 1 (espèces peu menacées) et 2 (espèces menacées) sont les plus représentés avec respectivement 71 et 41 espèces. Les espèces très menacées (niveau 3) sont au nombre de 18. Parmi celles-ci, Kigelia africana est l'espèce la plus menacée avec un score de 16 points. Au nombre des stratégies endogènes de conservation pratiquées, on note la conservation au champ (21 espèces concernées) et la conservation dans les jardins de case (34 espèces). 75 espèces ne bénéficient d'aucun mode de conservation. En définitive, l'étude a permis d'identifier les plantes antidiabétiques menacées, prioritaires pour la conservation.

(C) 2016 International Formulae Group. All rights reserved.

Mots clés : Gestion durable, enquêtes ethnobotaniques, plantes médicinales

\section{Vulnerability and endogenous strategies of conservation of the plants used in the treatment of diabetes in the townships of Glazoué and Savè in Benin center}

\section{ABSTRACT}

Excessive harvesting of organs of plants for medicinal purposes is a threat factor on phytodiversity. This survey achieved in the townships of Glazoué and Savè in Benin center, aimed at valuating the 
vulnerability and the endogenous strategies of conservation of antidiabetic plants. Indeed, 126 persons of which 35 women and 91 men have been investigated. The interviews were about the inventory of the antidiabetic plants; the different organs used and marketed and the endogenous strategies of conservation of the aforesaid plants. The Rapid Vulnerability Approach has been used. The results show that 144 plants are used in the traditional treatment of diabetes. Three levels of pressure have been distinguished. The levels 1 (little threatened species) and 2 (threatened species) are the more represented with respectively 71 and 41 species. The very threatened species (level 3) are up to 18, among which Kigelia africana is the most threatened, with a score of 16 among the endogenous strategies of conservation practised, there is the conservation in the field (with 21 species) and the conservation in the home gardens (34 species). 75 species don't benefit from any mode of conservation. Finally, the survey permitted to identify the threatened antidiabetic plants, important for the conservation.

(c) 2016 International Formulae Group. All rights reserved.

Keywords: Lasting management, ethnobotanical investigations, medicinal plants.

\section{INTRODUCTION}

L'usage traditionnel des plantes médicinales constitue la base de la médecine curative des populations à revenu modeste (Dounias et al., 2000) mais si l'on n'y prend garde, il constitue également l'une des causes de destruction des formations végétales (Makumbelo et al., 2008). C'est justement le cas des plantes médicinales fortement répandues dans les forêts et les savanes africaines et qui ont pendant plusieurs millénaires été utilisées comme le seul moyen de traitement par les peuples africains d'autrefois (Tchigankong, 2009). La connaissance des espèces vulnérables du fait de leur utilisation en phytothérapie traditionnelle est un grand pas vers une meilleure exploitation de ces ressources. Elle permet de savoir sur quelles espèces, devront être portées, de manière prioritaire, des mesures restrictives (Okou, 2009).

Au Bénin, 3000 plantes ont été inventoriées dans les écosystèmes forestiers (Anonyme, 2002). Parmi ces espèces de plantes identifiées, 814 sont consommées par les populations locales comme plantes médicinales (Sinsin et Owolabi, 2001) avec toutes ou presque toutes les parties quotidiennement utilisées (Geldenhuys et Delvaux, 2002; Zassada, 2003). Malheureusement, cette diversité spécifique s'appauvrit de nos jours à un rythme alarmant. $\mathrm{La}$ déforestation, les changements climatiques, la surexploitation, l'agriculture, la pression démographique, l'élevage, les feux de végétation, etc. contribuent à la perte des plantes indigènes (Assogbadjo et al., 2011). Il en ressort que la conservation des espèces médicinales africaines est capitale aussi bien pour le bien-être des populations que pour le développement de l'industrie pharmaceutique au niveau du continent. Mais, bien qu'il y ait eu d'importantes études relatives à l'identification de ces espèces (Adomou et al., 2012; Fah et al., 2013; Lalèyè et al., 2015; Lawin et al., 2015 ; Yapi et al., 2015), à leur valorisation par les populations locales et à leur composition biochimique (Fabricant et Farnsworth, 2001 ; Taylor et al., 2001 ; Fennell et al., 2004), très peu d'études se sont intéressées à l'évaluation des menaces qui pèsent sur ces espèces et les stratégies endogènes de leur conservation. Ce gap d'informations pourrait entraver les efforts de conservation durable de ces espèces au profit de leurs utilisateurs.

En matière d'évaluation de menace, plusieurs méthodes sont utilisées: celles de Cunningham (2001), Betti (2001), Dzerefos et al. (2001), Wagner et al. (2008), et Traoré et al. (2011). Ces études ont dans l'ensemble proposé des mesures d'aménagement ou actions de conservation mieux adaptées et qui devraient être considérées comme prioritaires pour ces espèces identifiées. D'après Betti (2001), l'estimation des indices de vulnérabilité permet de comparer les espèces utilisées en médecine traditionnelle entre elles, afin de sélectionner, au stade actuel de nos connaissances, les espèces potentiellement 
menacées. L'utilisation durable de certaines espèces végétales employées dans la médecine traditionnelle est possible, mais certaines méthodes de récolte sont préjudiciables à la survie et à la pérennité des espèces concernées : écorçage, mutilation, déracinement, etc. La présente étude repose sur la méthode de Cunningham (2001) et a pour objectif d'évaluer la vulnérabilité et les stratégies endogènes de conservation des espèces végétales intervenant dans le traitement du diabète dans les communes de Glazoué et Savè au Centre-Bénin.

\section{MATERIEL ET METHODES Milieu d'étude}

Le cadre d'étude, situé entre $7^{\circ} 42^{\prime}$ et 8 ${ }^{\circ} 30^{\prime}$ latitude Nord puis $2{ }^{\circ} 05^{\prime}$ et $2{ }^{\circ} 45^{\prime}$ longitude Est, est constitué des Communes de Glazoué et Savè dans le Département des Collines au Bénin. Les deux communes d'étude réunies comptent 18 arrondissements dont 10 à Glazoué et huit (08) à Savè (Figure 1). Zone de transition climatique entre le subéquatorial et le soudanien, le milieu d'étude a un climat soudano-guinéen. La pluviométrie moyenne annuelle est de 1100 mm et la température moyenne annuelle est de $27{ }^{\circ} \mathrm{C}$. La végétation de la zone d'étude est constituée des formations naturelles (forêts galeries, savane boisée, arborée et arbustive et des jachères) et des formations artificielles (plantations de tecks et d'anacardiers).

\section{Collecte des données ethnobotaniques}

Les entretiens qui ont eu lieu ont porté sur les menaces qui pèsent sur la survie des espèces et les stratégies endogènes de leur conservation. Ainsi, il a été procédé au rassemblement des informations sur les espèces: les organes utilisés, les pratiques traditionnelles de conservation, l'importance de la commercialisation, leur distribution et abondance. Pour accorder plus de fiabilité aux déclarations relatives à l'abondance locale d'une espèce, les enquêtés abritant l'espèce dans leurs champs, jardins de case ou habitations ont été identifiés lors des enquêtes ethnobotaniques et une visite de terrain a été effectuée dans chacun de ces sites (habitats) pour constater la présence effective de l'espèce.

\section{Analyse des données \\ Menaces pesant sur les espèces}

Pour évaluer les menaces qui pèsent sur les espèces, nous avons utilisé cinq critères ou indicateurs de menace (issus des informations recueillies lors des enquêtes ethnobotaniques) en s'inspirant de la méthode d' «Evaluation Rapide de la Vulnérabilité (ERV)» développée par Cunningham (2001) et des critères développés par Lejoly (2001). Trois niveaux de pressions ou menace ont été distingués à savoir : Niveau 1 : faible (espèces peu menacées); Niveau 2: élevé (espèces menacées) ; Niveau 3 : très élevé (espèces très menacées). Ainsi, lorsque le niveau de pression est très élevé, la note 3 est attribuée ; si c'est élevé, la note 2 est attribuée et s'il est faible la note 1 . Les indicateurs retenus ont été: les organes recherchés : les impacts de la récolte, sur un individu de plante vont évidemment varier selon l'organe récolté (Cunningham, 2001). Les organes sont classés, en fonction de leur importance pour la survie de la plante en: organes très préjudiciables: racines, plante entière, jeune pousse (note 3); organes préjudiciables: écorce de la tige et sève (note 2); organes peu ou pas préjudiciables : feuilles, fleurs, graines, noix, bulbes (note 1); le nombre d'organes recherchés : si la partie utilisée est un indice de vulnérabilité, le nombre de parties différentes recherchées sur une même plante l'est également (Delvaux et Sinsin, 2003). Ainsi on a 3 organes recherchés (note 3 ) ; 2 organes recherchés (note 2); 1 organe recherché (note 1); les pratiques traditionnelles de conservation et culture des espèces: elles réduisent la pression sur les espèces. Ainsi : pratiques de conservation non existantes et espèces non plantées : note 3 ; pratiques de conservation discriminatoire en faveur d'autres utilisations : note 2 ; pratiques de conservation excluant la destruction de l'espèce ou espèces plantées : note 1 ; -la distribution géographique et abondance : une espèce à aire restreinte est plus vulnérable qu'une espèce à aire large. De même, une espèce peu abondante, dispersée, clairsemée est plus vulnérable qu'une espèce commune (Lejoly, 2001). La répartition des espèces est 
appréciée à partir des travaux de Arbonnier (2009) et la distribution locale des espèces dans la zone d'étude. A ce sujet, on a : espèces rares ou peu abondantes/peu communes : note 3 ; espèces abondantes/communes : note 2 ; espèces très abondantes/très communes : note 1 . L'estimation de l'abondance d'une espèce a été faite à partir du nombre d'enquêtés abritant ladite espèce dans son champ, jardin de case ou habitation. Si l'espèce est présente chez moins de $50 \%$ des enquêtés, elle est considérée comme rare ou peu abondante/peu commune ; $50 \%$ des enquêtés, elle est dite abondante/commune; plus de $50 \%$ des enquêtés, elle est dite très abondante/très commune ; l'importance de l'utilisation: la commercialisation d'une espèce en système formel ou informel la rend plus vulnérable (Lejoly, 2001). L'indicateur utilisé ici est le nombre de marchands de plantes médicinales enquêtés qui commercialisent l'espèce. Ainsi, lorsque plus de la moitié des marchands de plantes médicinales enquêtés commercialisent l'espèce, la note 3 est attribuée ; moins de la moitié les commercialisent, la note 2 ; aucun marchand de plantes médicinales enquêté ne commercialise l'espèce, la note 1 est attribuée.

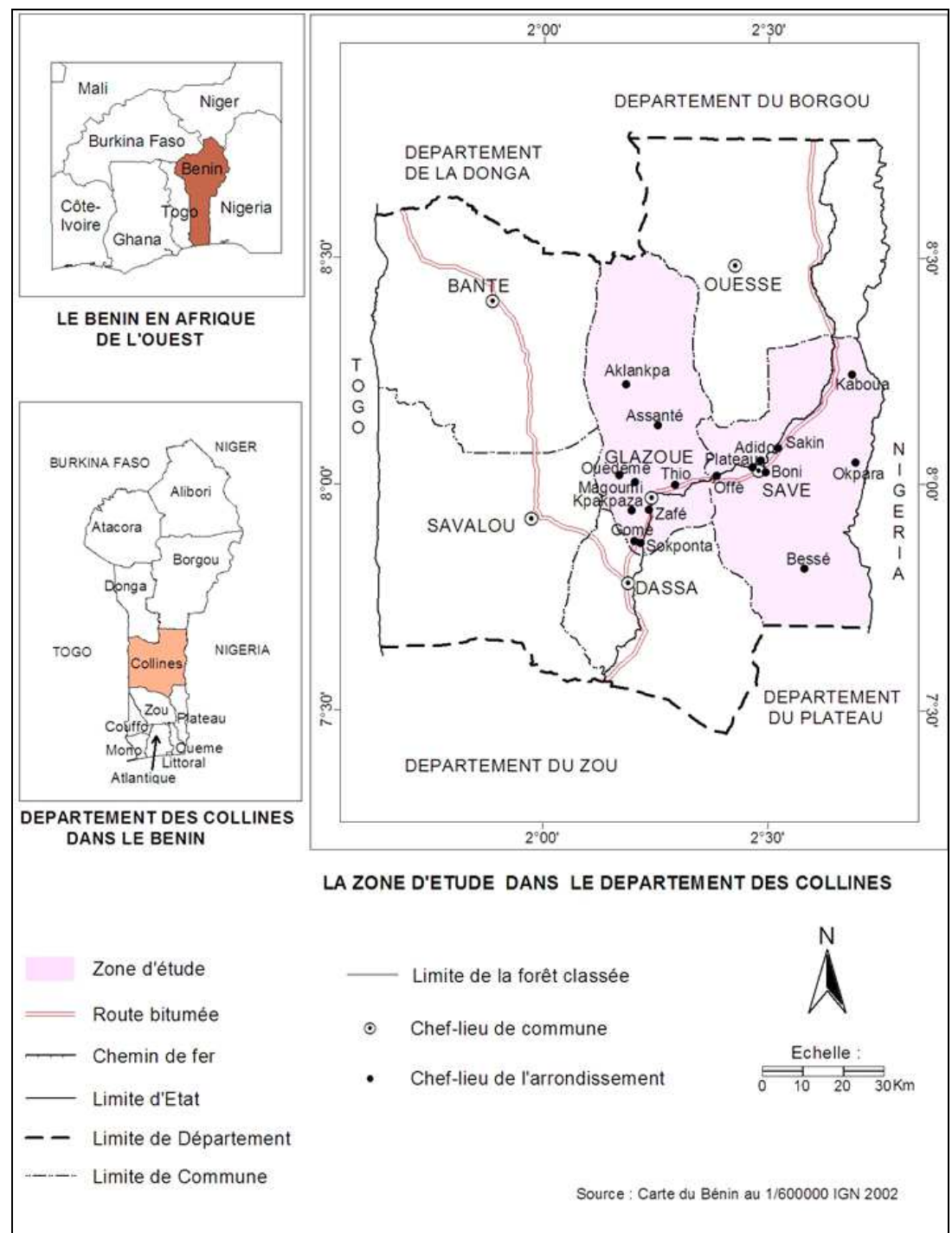

Figure 1: Situation géographique de la zone d'étude. 
Pour évaluer les différents niveaux de pression sur les espèces, il a été procédé à la sommation des scores de tous les critères pour chaque espèce. Ainsi, selon le score de chaque espèce, il a été distingué trois niveaux de pression ou menace à savoir : Niveau 1 (score $\leq 10)$ : espèces peu menacées ; Niveau 2 (10 < score < 13): espèces menacées; Niveau 3 (score $\geq 13)$ : espèces très menacées.

\section{Stratégies endogènes de conservation des espèces}

Les modes de conservation adoptés par les populations enquêtées ont pu être identifiés grâce aux données collectées. Ainsi, quatre modes de conservation des espèces ont été distingués: conservation au champ: ce sont les espèces épargnées aux champs ; conservation dans les jardins de case : il s'agit des espèces plantées ou laissées dans les jardins de cases/habitations/abords des maisons; conservation mixte: ce mode de conservation se dit pour une espèce qui est à la fois épargnée au champ et présente dans les jardins de case ; aucun : c'est pour les espèces qui ne remplissent aucune des conditions sus citées.

\section{RESULTATS \\ Menaces pesant sur les espèces}

L'évaluation des menaces qui pèsent sur les espèces a permis de distinguer trois niveaux de pression ou menace à savoir : Niveau 1 (espèces peu menacées), Niveau 2 (espèces menacées) et Niveau 3 (espèces très menacées) (Tableau 1). De l'analyse du Tableau 1, on constate que le niveau 1 est le plus représenté avec 71 espèces soit 54,61\% suivi du niveau 2 avec 41 espèces soit 31,53 $\%$ et du niveau 3 avec 18 espèces $(13,84 \%)$. Kigelia africana se révèle comme l'espèce la plus menacée avec un score de 16 points (Tableau 2).

\section{Stratégies endogènes de conservation des espèces \\ Les espèces végétales antidiabétiques} utilisées dans la zone d'étude bénéficient des stratégies endogènes de conservation (Tableau 3). De l'analyse du Tableau 3, il ressort que les espèces bénéficiant d'une conservation au champ sont très peu nombreuses $(16,15 \%)$. Par contre, celles ne jouissant d'aucun mode de conservation sont majoritaires $(57,69 \%)$. Globalement, les plantes sont plus conservées dans les jardins de case soit $26,15 \%$.

Tableau 1: Evaluation des menaces pesant sur les espèces.

\begin{tabular}{ll}
\hline $\begin{array}{c}\text { Niveaux de } \\
\text { pression }\end{array}$ & Espèces végétales \\
\hline & Abrus precatorius L.; Acanthospermum hispidum DC.Aloe buettneri A. Berger ; \\
& Anchomanes dalzielii N. E. Br.; Anogeissus leiocarpa (DC.) Guill. \& Perr. ; \\
& Azadirachta indica A. Juss. ; Blighia sapida König ; Borassus aethiopum Mart.; \\
& Brassica oleracea L.; Caesalpinia pulcherrima (L.) Sw.; Calotropis procera \\
& (Aiton) W. T. Aiton ; Capsicum frutescens L. ; Carica papaya L. ; \\
& Catharanthus roseus (L.) G. Don ; Citrullus colocynthis (L.) Schrad. ; \\
& Combretum collinum Fresen.; Corchorus olitorius L. ; Crateva adansonii DC. \\
& Ssp. Adansonii ; Croton gratissimus Bureh.; Cussonia arborea Hochst. ex A. \\
& Rich.; Cymbopogon citratus (DC.) Stapf ; Cymbopogon giganteus (Hochst.) \\
& Chiov. ; Daucus carota L. ssp. sativus (Hoffm.) Arcang.; Dioscorea cayenensis \\
& Lam.; Elaeis guineensis Jacq. ; Erythrina senegalensis DC. ; Euphorbia \\
& hyssopifolia L.; Ficus platyphylla Delile ; Ficus sycomorus L. ; Ficus umbellata \\
& Vahl ; Gladiolus daleii van Geel ; Glycine max (L.) Merr. ; Gossypium \\
& arboreum L. ; Heliotropium indicum L. ; Isoberlinia doka Craib \& Stapf; \\
& Jatropha gossypiifolia L. ; Lannea barteri (Oliv.) Engl. ; Manihot esculenta \\
& Crantz; Merremia quinquefolia (L.) Hallier f. ;Milicia excelsa (Welw.) \\
\hline
\end{tabular}


C.C.Berg; Mitracarpus villosus (Sw.) DC. ; Momordica charantia L. ; Moringa oleifera Lam.; Musa paradisiaca L.; Nicotiana tabacum L.; Ocimum gratissimum L.; Opilia amentacea Roxb.; Pavetta crassipes K.Schum.; Pericopsis laxiflora (Benth. ex Baker) Meeuwen; Persea americana Mill.; Phaseolus lunatus L.; Phyllanthus amarus Schumach. \& Thonn; Psidium guajava L.; Raphionacme brownii Scott-Elliot ; Saccharum officinarum L.; Schrankia leptocarpa DC.; Secamone afzelii (Schult.) K.Schum.; Sesamum radiatum Schumach. \& Thonn.; Spondias mombin L.; Sterculia setigera Delile; Stereospermum kunthianum Cham.; Tacca leontopetaloides (L.) Kuntze; Tamarindus indica L.; Vernonia amygdalina Delile; Vernonia cinerea (L.) Less.; Vitellaria paradoxa C.F.Gaertn. ssp. Paradoxa ; Zea mays L.

Acmella uliginosa (Sw.) Cass.; Anacardium occidentale L. ;Ananas comosus (L.) Merr. ; Anthocleista vogelii Planch. ; Bridelia ferruginea Benth.; Carissa spinarum L.; Chromolaena odorata (L.) R. M. King; Citrus aurantifolia (Christm. \& Panzer) Swingle; Commelina erecta L. ssp. erecta; Crinum zeylanicum (L.) L.; Crotalaria retusa L.; Curculigo pilosa (Schumach. \& Thonn.) Engl.;Daniellia oliveri (Rolfe) Hutch. \& Dalziel; Euphorbia hirta L. ;Gardenia erubescens Stapf \& Hutch. ;Gymnosporia senegalensis (Lam.) Loes.;Hymenocardia acida Tul. ;Hyptis pectinata (L.) Poit. ;Jatropha curcas L. ; Lannea acida A. Rich. s. 1.; Lantana camara L.;Mangifera indica L.;Morelia senegalensis A.Rich. ex DC.; Newbouldia laevis (P.Beauv.) Seemann ex Bureau;Ocimum americanum L. Omphalogonus calophyllus Baill. ;Parinari curatellifolia Planch. ex Benth.; Paullinia pinnata L. ;Philenoptera cyanescens (Schumach. \& Thonn.) Roberty; Plumbago zeylanica L.; Pseudocedrela kotschyi (Schweinf.) Harms ;Pteleopsis suberosa Engl. \& Diels ; Rauvolfia vomitoria Afzel.; Rhynchosia pycnostachya (DC.) Meikle ;Rourea coccinea (Thonn. ex Schumach.) Benth.;Saba comorensis (Boj.) Pichon;Senna siamea (Lam.) H.S.Irwin \& Barneby; Strophanthus hispidus DC.; Swartzia madagascariensis Desv.; Tetrapleura tetraptera (Schumach. \& Thonn.) Taub.; Trichilia emeticaVahl;Uvaria chamae P. Beauv.; Vangueriella spinosa (Schumach. \& Thonn.) Verdc.; Zanthoxylum zanthoxyloides (Lam.) Zepernick \& Timler; Zingiber officinale Roscoe

Annona senegalensis Pers. ; Aristolochia albida Duch. ; Burkea africana Hook.; Caesalpinia bonduc (L.) Roxb.; Cocos nucifera L. ;Detarium microcarpum Guill. \& Perr.; Eriosema glomeratum (Guill. \& Perr.) Hook.f. var. glomeratum; Flacourtia indica (Burm.f.) Merr.; Flueggea virosa (Roxb.ex

3 Willd) Voigt.; Imperata cylindrica (L.) P. Beauv.; Khaya senegalensis (Desr.) A. Juss.; Kigelia africana (Lam.) Benth.; Morinda lucida Benth.; Olax subscorpioidea Oliv. ; Oxytenanthera abyssinica (A.Rich.) Munro ; Sansevieria liberica hort. ex Gerome \& Labroy ; Sarcocephalus latifolius (Sm.) E.A.Bruce; Securidaca longepedunculata Fresen. ; 


\section{F. LAWIN et al. / Int. J. Biol. Chem. Sci. 10(3): 1069-1085, 2016}

Tableau 2: Critères d'évaluation des menaces pesant sur les espèces.

\begin{tabular}{|c|c|c|c|c|c|c|c|}
\hline \multirow[b]{2}{*}{$\mathbf{N}^{\circ}$} & \multirow[b]{2}{*}{ ESPECES } & \multicolumn{5}{|c|}{ Indicateurs (critères) } & \multirow[b]{2}{*}{ Score } \\
\hline & & $\mathbf{A}$ & $\mathbf{B}$ & $\mathbf{C}$ & $\mathbf{D}$ & $\mathbf{E}$ & \\
\hline 1 & Abrus precatorius $\mathrm{L}$. & 1 & 1 & 1 & 3 & 2 & 8 \\
\hline 2 & Acanthospermum hispidum DC. & 1 & 1 & 3 & 3 & 1 & 9 \\
\hline 3 & Acmella uliginosa (Sw.) Cass. & 3 & 1 & 3 & 3 & 1 & 11 \\
\hline 4 & Aframomum melegueta (Roscoe) K. Schum. & - & - & - & - & - & - \\
\hline 5 & Allium ascalonicum auct. non Strand & - & - & - & & - & - \\
\hline 6 & Allium сера $\mathrm{L}$ & - & & - & & - & - \\
\hline 7 & Allium sativum $\mathrm{L}$. & - & & - & & - & - \\
\hline 8 & Aloe buettneri A. Berger & 1 & 1 & 1 & 3 & 1 & 7 \\
\hline 9 & Anacardium occidentale L. & 6 & 3 & 1 & 1 & 1 & 12 \\
\hline 10 & Ananas comosus (L.) Merr. & 4 & 2 & 1 & 3 & 1 & 11 \\
\hline 11 & Anchomanes dalzielii N. E. Br. & 1 & 2 & 3 & 3 & 1 & 10 \\
\hline 12 & Annona senegalensis Pers. & 4 & 2 & 3 & 3 & 1 & 13 \\
\hline 13 & Anogeissus leiocarpa (DC.) Guill. \& Perr. & 2 & 1 & 3 & 3 & 1 & 10 \\
\hline 14 & Anthocleista vogelii Planch. & 3 & 1 & 3 & 3 & 1 & 11 \\
\hline 15 & Aristolochia albida Duch. & 3 & 1 & 3 & 3 & 3 & 13 \\
\hline 16 & Azadirachta indica A. Juss. & 3 & 2 & 1 & 2 & 1 & 9 \\
\hline 17 & Blighia sapida König & 3 & 3 & 1 & 2 & 1 & 10 \\
\hline 18 & Borassus aethiopum Mart. & 3 & 1 & 1 & 2 & 1 & 8 \\
\hline 19 & Brassica oleracea L. & 1 & 1 & 1 & 3 & 1 & 7 \\
\hline 20 & Bridelia ferruginea Benth. & 3 & 2 & 3 & 3 & 1 & 12 \\
\hline 21 & Burkea africana Hook. & 5 & 2 & 3 & 3 & 1 & 14 \\
\hline 22 & Caesalpinia bonduc (L.) Roxb. & 4 & 2 & 1 & 3 & 3 & 13 \\
\hline 23 & Caesalpinia pulcherrima (L.) Sw. & 1 & 1 & 1 & 3 & 1 & 7 \\
\hline 24 & Calotropis procera (Aiton) W. T. Aiton & 4 & 2 & 1 & 2 & 1 & 10 \\
\hline
\end{tabular}


I. F. LAWIN et al. / Int. J. Biol. Chem. Sci. 10(3): 1069-1085, 2016

\begin{tabular}{|c|c|c|c|c|c|c|c|}
\hline 25 & Cannabis sativa $\mathrm{L}$. & - & - & - & - & - & - \\
\hline 26 & Capsicum frutescens $\mathrm{L}$. & 1 & 1 & 1 & 1 & 2 & 6 \\
\hline 27 & Carica papaya $\mathrm{L}$. & 4 & 3 & 1 & 1 & 1 & 10 \\
\hline 28 & Carissa spinarum $\mathrm{L}$. & 3 & 1 & 3 & 3 & 1 & 11 \\
\hline 29 & Catharanthus roseus (L.) G. Don & 1 & 1 & 1 & 3 & 1 & 7 \\
\hline 30 & Chromolaena odorata (L.) R. M. King & 3 & 1 & 3 & 3 & 1 & 11 \\
\hline 31 & Citrullus colocynthis (L.) Schrad. & 1 & 1 & 1 & 1 & 1 & 5 \\
\hline 32 & Citrus aurantifolia (Christm. \& Panzer) Swingle & 4 & 3 & 1 & 2 & 1 & 11 \\
\hline 33 & Cocos nucifera $\mathrm{L}$. & 6 & 3 & 1 & 3 & 1 & 14 \\
\hline 34 & Cola acuminata (P. Beauv.) Schott \& Endl. & - & - & - & - & - & - \\
\hline 35 & Cola nitida (Vent.) Schott \& Endl. & - & - & - & - & - & - \\
\hline 36 & Combretum collinum Fresen. & 1 & 1 & 3 & 3 & 1 & 9 \\
\hline 37 & Commelina erecta L. ssp. Erecta & 3 & 1 & 3 & 3 & 1 & 11 \\
\hline 38 & Corchorus olitorius $\mathrm{L}$. & 1 & 1 & 1 & 3 & 1 & 7 \\
\hline 39 & Crateva adansonii DC. ssp. Adansonii & 1 & 1 & 3 & 3 & 1 & 9 \\
\hline 40 & Crinum zeylanicum (L.) L. & 4 & 2 & 1 & 3 & 1 & 11 \\
\hline 41 & Crotalaria retusa $\mathrm{L}$. & 3 & 1 & 3 & 3 & 1 & 11 \\
\hline 42 & Croton gratissimus Bureh. & 1 & 1 & 1 & 3 & 2 & 8 \\
\hline 43 & Curculigo pilosa (Schumach. \& Thonn.) Engl. & 1 & 1 & 3 & 3 & 3 & 11 \\
\hline 44 & Cussonia arborea Hochst. ex A. Rich. & 1 & 1 & 1 & 3 & 1 & 7 \\
\hline 45 & Cymbopogon citratus (DC.) Stapf & 1 & 1 & 1 & 3 & 1 & 7 \\
\hline 46 & Cymbopogon giganteus (Hochst.) Chiov. & 1 & 1 & 3 & 3 & 1 & 9 \\
\hline 47 & Daniellia oliveri (Rolfe) Hutch. \& Dalziel & 5 & 2 & 1 & 2 & 1 & 11 \\
\hline 48 & Daucus carota L. ssp. sativus (Hoffm.) Arcang. & 3 & 1 & 1 & 3 & 1 & 9 \\
\hline 49 & Detarium microcarpum Guill. \& Perr. & 5 & 2 & 3 & 3 & 1 & 14 \\
\hline 50 & Dioscorea cayenensis Lam. & 1 & 1 & 1 & 1 & 1 & 5 \\
\hline 51 & Elaeis guineensis Jacq. & 1 & 1 & 1 & 2 & 1 & 6 \\
\hline 52 & Eriosema glomeratum (Guill. \& Perr.) Hook.f. var. Glomeratum & 4 & 2 & 3 & 3 & 1 & 13 \\
\hline
\end{tabular}


I. F. LAWIN et al. / Int. J. Biol. Chem. Sci. 10(3): 1069-1085, 2016

\begin{tabular}{|c|c|c|c|c|c|c|c|}
\hline 53 & Erythrina senegalensis DC. & 3 & 1 & 1 & 3 & 1 & 9 \\
\hline 54 & Eugenia aromatica (L.) Baill. & - & - & - & - & - & - \\
\hline 55 & Euphorbia hirta L. & 3 & 1 & 3 & 3 & 1 & 11 \\
\hline 56 & Euphorbia hyssopifolia L. & 1 & 1 & 3 & 3 & 1 & 9 \\
\hline 57 & Ficus platyphylla Delile & 2 & 1 & 3 & 3 & 1 & 10 \\
\hline 58 & Ficus sycomorus L. & 2 & 1 & 3 & 3 & 1 & 10 \\
\hline 59 & Ficus umbellata Vahl & 1 & 1 & 1 & 1 & 1 & 5 \\
\hline 60 & Flacourtia indica (Burm.f.) Merr. & 4 & 2 & 3 & 3 & 1 & 13 \\
\hline 61 & Flueggea virosa (Roxb.ex Willd) Voigt. & 4 & 2 & 3 & 3 & 1 & 13 \\
\hline 62 & Garcinia kola Heckel & - & - & - & - & - & - \\
\hline 63 & Gardenia erubescens Stapf \& Hutch. & 3 & 1 & 3 & 3 & 2 & 12 \\
\hline 64 & Gladiolus daleii van Geel & 1 & 1 & 3 & 3 & 1 & 9 \\
\hline 65 & Glycine $\max (\mathrm{L}$.$) Merr.$ & 1 & 1 & 1 & 1 & 1 & 5 \\
\hline 66 & Gossypium arboreum $\mathrm{L}$. & 1 & 1 & 3 & 3 & 1 & 9 \\
\hline 67 & Gymnosporia senegalensis (Lam.) Loes. & 3 & 1 & 3 & 3 & 1 & 11 \\
\hline 68 & Heliotropium indicum $\mathrm{L}$. & 1 & 1 & 1 & 3 & 1 & 7 \\
\hline 69 & Hymenocardia acida Tul. & 3 & 1 & 3 & 3 & 1 & 11 \\
\hline 70 & Hyptis pectinata (L.) Poit. & 3 & 1 & 3 & 3 & 1 & 11 \\
\hline 71 & Imperata cylindrica (L.) P. Beauv. & 4 & 2 & 3 & 3 & 1 & 13 \\
\hline 72 & Isoberlinia doka Craib \& Stapf & 1 & 1 & 3 & 3 & 1 & 9 \\
\hline 73 & Jatropha curcas L. & 4 & 2 & 1 & 3 & 1 & 11 \\
\hline 74 & Jatropha gossypiifolia L. & 1 & 1 & 1 & 3 & 1 & 7 \\
\hline 75 & Khaya senegalensis (Desr.) A. Juss. & 5 & 2 & 1 & 2 & 3 & 13 \\
\hline 76 & Kigelia africana (Lam.) Benth. & 6 & 3 & 3 & 3 & 1 & 16 \\
\hline 77 & Lannea acida A. Rich. s. 1. & 2 & 1 & 3 & 3 & 2 & 11 \\
\hline 78 & Lannea barteri (Oliv.) Engl. & 2 & 1 & 3 & 3 & 1 & 10 \\
\hline 79 & Lantana camara $\mathrm{L}$ & 3 & 1 & 3 & 3 & 1 & 11 \\
\hline 80 & Mangifera indica $\mathrm{L}$. & 6 & 3 & 1 & 1 & 1 & 12 \\
\hline
\end{tabular}


I. F. LAWIN et al. / Int. J. Biol. Chem. Sci. 10(3): 1069-1085, 2016

Manihot esculenta Crantz

$\begin{array}{lllllll}1 & 1 & 1 & 1 & 1 & 5\end{array}$

Merremia quinquefolia (L.) Hallier f.

$1 \quad 1 \quad 3 \quad 3$

$\begin{array}{llll}2 & 1 & 1 & 3\end{array}$

$\begin{array}{llll}1 & 1 & 3 & 3\end{array}$

Mitracarpus villosus (Sw.) DC.

Momordica charantia L.

7

Monodora myristica (Gaertn.) Dunal

$\begin{array}{llll}1 & 1 & 1 & 3 \\ - & - & - & -\end{array}$

$\begin{array}{llll}3 & 1 & 3 & 3\end{array}$

Morelia senegalensis A.Rich. ex DC.

$\begin{array}{llll}6 & 3 & 1 & 3\end{array}$

Morinda lucida Benth.

Moringa oleifera Lam.

$\begin{array}{llll}1 & 2 & 1 & 3\end{array}$

Musa paradisiaca $\mathrm{L}$

Newbouldia laevis (P.Beauv.) Seemann ex Bureau

$\begin{array}{llll}1 & 1 & 3 & 3\end{array}$

$\begin{array}{llll}3 & 2 & 2 & 3\end{array}$

Nicotiana tabacum $\mathrm{L}$.

Ocimum americanum $\mathrm{L}$.

Ocimum gratissimum $\mathrm{L}$.

Olax subscorpioidea Oliv.

Omphalogonus calophyllus Baill.

Opilia amentacea Roxb.

Oxytenanthera abyssinica (A.Rich.) Munro

Parinari curatellifolia Planch. ex Benth.

Paullinia pinnata $\mathrm{L}$.

Pavetta crassipes K.Schum.

Pennisetum americanum (L.) Leeke

Pericopsis laxiflora (Benth. ex Baker) Meeuwen

Persea americana Mill.

Phaseolus lunatus $\mathrm{L}$.

Philenoptera cyanescens (Schumach. \& Thonn.) Roberty

$\begin{array}{llll}1 & 1 & 1 & 3\end{array}$

$\begin{array}{llll}4 & 2 & 1 & 3\end{array}$

$\begin{array}{llll}1 & 1 & 1 & 3\end{array}$

$\begin{array}{llll}3 & 1 & 3 & 3\end{array}$

$\begin{array}{llll}3 & 1 & 3 & 3\end{array}$

$\begin{array}{llll}1 & 1 & 3 & 3\end{array}$

$\begin{array}{llll}4 & 2 & 3 & 3\end{array}$

$\begin{array}{llll}5 & 2 & 1 & 3\end{array}$

$\begin{array}{llll}4 & 2 & 1 & 3\end{array}$

$\begin{array}{llll}1 & 1 & 3 & 3\end{array}$

$\begin{array}{llll}1 & 1 & 3 & 3\end{array}$

$\begin{array}{llll}1 & 1 & 1 & 3\end{array}$

$\begin{array}{llll}1 & 1 & 1 & 3\end{array}$

$\begin{array}{llll}3 & 1 & 3 & 3\end{array}$

$\begin{array}{llll}3 & 1 & 1 & 3\end{array}$ 
I. F. LAWIN et al. / Int. J. Biol. Chem. Sci. 10(3): 1069-1085, 2016

\begin{tabular}{|c|c|c|c|c|c|c|c|}
\hline 109 & Piper guineense Schumach. \& Thonn. & - & - & - & - & - & - \\
\hline 110 & Plumbago zeylanica $\mathrm{L}$. & 4 & 2 & 1 & 3 & 1 & 11 \\
\hline 111 & Pseudocedrela kotschyi (Schweinf.) Harms & 3 & 1 & 3 & 3 & 1 & 11 \\
\hline 112 & Psidium guajava $\mathrm{L}$. & 4 & 2 & 1 & 2 & 1 & 10 \\
\hline 113 & Pteleopsis suberosa Engl. \& Diels & 3 & 2 & 3 & 3 & 1 & 12 \\
\hline 114 & Raphionacme brownii Scott-Elliot & 1 & 1 & 3 & 3 & 1 & 9 \\
\hline 115 & Rauvolfia vomitoria Afzel. & 3 & 1 & 3 & 3 & 1 & 11 \\
\hline 116 & Rhynchosia pycnostachya (DC.) Meikle & 3 & 1 & 3 & 3 & 1 & 11 \\
\hline 117 & Rourea coccinea (Thonn. ex Schumach.) Benth. & 3 & 1 & 3 & 3 & 1 & 11 \\
\hline 118 & Saba comorensis (Boj.) Pichon & 3 & 1 & 3 & 3 & 1 & 11 \\
\hline 119 & Saccharum officinarum $\mathrm{L}$. & 4 & 2 & 1 & 2 & 1 & 10 \\
\hline 120 & Sansevieria liberica hort. ex Gerome \& Labroy & 4 & 3 & 3 & 3 & 1 & 14 \\
\hline 121 & Sarcocephalus latifolius (Sm.) E.A.Bruce & 3 & 1 & 3 & 3 & 3 & 13 \\
\hline 122 & Schrankia leptocarpa DC. & 1 & 1 & 1 & 3 & 1 & 7 \\
\hline 123 & Secamone afzelii (Schult.) K.Schum. & 1 & 1 & 3 & 3 & 1 & 9 \\
\hline 124 & Securidaca longepedunculata Fresen. & 3 & 1 & 3 & 3 & 3 & 13 \\
\hline 125 & Senna siamea (Lam.) H.S.Irwin \& Barneby & 5 & 2 & 3 & 3 & 1 & 12 \\
\hline 126 & Sesamum radiatum Schumach. \& Thonn. & 1 & 1 & 3 & 3 & 1 & 9 \\
\hline 127 & Spondias mombin L. & 3 & 2 & 1 & 3 & 1 & 10 \\
\hline 128 & Sterculia setigera Delile & 2 & 1 & 1 & 3 & 1 & 8 \\
\hline 129 & Stereospermum kunthianum Cham. & 2 & 1 & 3 & 3 & 1 & 10 \\
\hline 130 & Strophanthus hispidus DC. & 3 & 1 & 3 & 3 & 1 & 11 \\
\hline 131 & Swartzia madagascariensis Desv. & 3 & 1 & 3 & 3 & 1 & 11 \\
\hline 132 & Tacca leontopetaloides (L.) Kuntze & 1 & 1 & 3 & 3 & 1 & 9 \\
\hline 133 & Tamarindus indica $\mathrm{L}$ & 3 & 2 & 1 & 3 & 1 & 10 \\
\hline 134 & Tetrapleura tetraptera (Schumach. \& Thonn.) Taub. & 1 & 1 & 3 & 3 & 3 & 11 \\
\hline 135 & Trichilia emetica Vahl & 3 & 1 & 3 & 3 & 1 & 11 \\
\hline 136 & Uvaria chamae P. Beauv. & 3 & 1 & 1 & 3 & 3 & 11 \\
\hline
\end{tabular}


I. F. LAWIN et al. / Int. J. Biol. Chem. Sci. 10(3): 1069-1085, 2016

\begin{tabular}{|c|c|c|c|c|c|c|c|}
\hline 137 & Vangueriella spinosa (Schumach. \& Thonn.) Verdc. & 3 & 1 & 3 & 3 & 1 & 11 \\
\hline 138 & Vernonia amygdalina Delile & 4 & 2 & 1 & 1 & 1 & 9 \\
\hline 139 & Vernonia cinerea (L.) Less. & 1 & 1 & 3 & 3 & 1 & 9 \\
\hline 140 & Vitellaria paradoxa C.F.Gaertn. ssp. Paradoxa & 2 & 1 & 1 & 2 & 1 & 7 \\
\hline 141 & Xylopia aethiopica (Dunal) A. Rich. & - & - & - & - & - & - \\
\hline 142 & Zanthoxylum zanthoxyloides (Lam.) Zepernick \& Timler & 3 & 1 & 3 & 3 & 2 & 12 \\
\hline 143 & Zea mays L. & 1 & 1 & 1 & 1 & 1 & 5 \\
\hline 144 & Zingiber officinale Roscoe & 3 & 1 & 1 & 3 & 3 & 11 \\
\hline
\end{tabular}

NB : Les 14 plantes importées ou qui ne se cultivent pas dans le milieu d'étude (Aframomum melegueta, Allium ascalonicum, Allium cepa, Allium sativum, Cannabis sativa., Cola acuminata, Cola nitida, Eugenia aromatica, Garcinia kola, Monodora myristica, Pennisetum americanum, Picralima nítida, Piper guineense et Xylopia aethiopica) d'après nos enquêtes n'ont pas été prises en compte dans l'évaluation du fait que tous les critères ne peuvent leur être appliqués. C'est le cas des deux critères à savoir: «pratiques traditionnelles de conservation et culture des espèces » et « distribution géographique et abondance ». .C'est seulement 130 plantes qui ont fait l'objet d'évaluation. A= Organes recherchés

B= Nombre d'organes recherchés

$\mathbf{C}=$ Pratiques traditionnelles de conservation et culture des espèces

D= Distribution géographique et Abondance

$\mathbf{E}=$ Importance de l'utilisation

Tableau 3: Stratégies endogènes de conservation des espèces.

\begin{tabular}{|c|c|c|c|}
\hline $\begin{array}{c}\text { Modes de } \\
\text { conservation }\end{array}$ & Espèces végétales & $\mathbf{N}$ & $\%$ \\
\hline Champ (1) & $\begin{array}{l}\text { Azadirachta indica A. Juss.; Blighia sapida König; Borassus aethiopum Mart.; Calotropis procera (Aiton) W. T. Aiton ; } \\
\text { Carica papaya L.; Cocos nucifera L.; Cussonia arborea Hochst. ex A. Rich.; Daniellia oliveri (Rolfe) Hutch. \& Dalziel; } \\
\text { Erythrina senegalensis DC.; Mangifera indica L.; Milicia excelsa (Welw.) C.C.Berg; Momordica charantia L.; Ocimum } \\
\text { gratissimum L.; Parinari curatellifolia Planch. ex Benth.; Paullinia pinnata L.; Psidium guajava L.; Spondias mombin L ; } \\
\text { Sterculia setigera Delile; Tamarindus indica L.; Uvaria chamae P. Beauv.; Vitellaria paradoxa C.F.Gaertn. ssp. paradoxa. }\end{array}$ & 21 & 16,15 \\
\hline Jardin de case (2) & $\begin{array}{l}\text { Abrus precatorius L.; Aloe buettneri A. Berger; Azadirachta indica A. Juss.; Blighia sapida König; Caesalpinia bonduc (L.) } \\
\text { Roxb.; Caesalpinia pulcherrima (L.) Sw; Calotropis procera (Aiton) W. T. Aiton; Carica papaya L.; Catharanthus roseus } \\
\text { (L.) G. Don; Citrus aurantifolia (Christm. \& Panzer) Swingle; Cocos nucifera L.; Crateva adansonii DC. ssp. Adansonii; }\end{array}$ & 34 & 26,15 \\
\hline
\end{tabular}




\section{DISCUSSION}

Menaces pesant sur les espèces

L'évaluation des menaces pesant sur les espèces a révélé 18 plantes très menacées sur 130. Trois (03) plantes sur les 18 sont inscrites sur la liste rouge de l'IUCN (Union Internationale pour la Conservation de la Nature) pour le Bénin établie par Adomou et al. (2011). Il s'agit de $C$. bonduc, $K$. senegalensis et $K$. africana. Wagner et al. (2008) ont rapporté 7 espèces potentiellement menacées sur 102 plantes étudiées au Népal. Sigdel et Rokaya (2011) ont, quant à eux, fait mention de 9 plantes très menacées sur 85 dans le même pays. Utilisant la même méthode au Cameroun, Betti (2001) a signalé quatre espèces très vulnérables sur 23 plantes antipaludiques. La méthode d'évaluation rapide de vulnérabilité permet donc d'appréhender les espèces les plus menacées, au sein d'un groupe de plantes, renseignant ainsi sur celles prioritaires pour la conservation. De l'évaluation, $K$. africana ayant le score (16) le plus élevé, est l'espèce la plus menacée. Ce résultat concorde avec les travaux de Adomou (2005) qui indiquent que $K$. africana est l'une des 280 espèces végétales menacées du Bénin et de Dassou et al. (2014) qui ont signalé la même espèce parmi les 31 plantes vulnérables à usages vétérinaires au Nord-Bénin. De même, ce résultat corrobore celui mentionné par Okou (2009) dans une étude sur la vulnérabilité des ressources forestières médicinales du noyau central de la forêt classée de la Lama au SudBénin face aux prélèvements ethnobotaniques; ce qui s'explique par une forte sollicitation de ses organes (racine, écorce, fruit) et sa rareté dans le milieu.

Notons que les menaces pesant sur les plantes cultivées citées dans cette étude sont négligeables du fait que ces plantes sont renouvelées régulièrement par l'homme.

\section{Stratégies endogènes de conservation des espèces}

Par rapport aux stratégies endogènes de conservation, seulement $26,15 \%$ des espèces bénéficient d'une conservation dans les jardins de case. Ce qui veut dire que les tentatives de domestication des espèces recensées sont faibles. Or, l'utilisation fréquente d'une espèce végétale sans aucune tentative de domestication entraîne l'augmentation de sa vulnérabilité. A la longue, on assistera à une disparition de spécimens de végétaux provoquée par les cueillettes excessives et les pressions humaines croissantes (Delvaux et Sinsin, 2003; Keirungi et Fabricius, 2005). Cependant, les plantes sont plus conservées dans les jardins de case qu'aux champs. Ce qui témoigne de la contribution des jardins de case à la conservation de la biodiversité au Bénin comme précédemment illustré dans plusieurs travaux (Salako et al., 2014 ; Idohou et al., 2014). De même, ce résultat s'accorde à ceux de Edward et Kabir (2009); Galluzzi et al. (2010) qui ont indiqué que les jardins de case constituent des réservoirs de la diversité biologique. Les plantes des jardins de case sont utilisées à des fins alimentaires et médicinales (Souto et Ticktin, 2012 ; Idohou et al., 2014). C'est le cas par exemple de Carica papaya L., Citrus aurantifolia (Christm. \& Panzer) Swingle; Cocos nucifera L., Mangifera indica L., Spondias mombin L. et Vernonia amygdalina Delile rapportés dans cette étude qui est utilisés non seulement pour l'alimentation mais aussi pour soigner le diabète.

\section{Conclusion}

Cette étude a permis d'identifier les plantes antidiabétiques prioritaires pour la conservation. L'analyse des informations recueillies révèle que certaines plantes sont utilisées pour leurs feuilles, leurs racines, leurs écorces, leurs graines, leurs fruits et fleurs alors que d'autres sont utilisées entièrement. Le prélèvement de nombreux organes des plantes médicinales cause la destruction des pieds de certaines espèces et accentue la dégradation des écosystèmes. Des efforts de domestication des spécimens sauvages demeurent donc une réalité. $K$ africana étant l'espèce la plus menacée, elle 
mérite des études plus approfondies en vue de sa domestication et sa conservation au Bénin.

\section{CONFLIT D'INTERETS}

Les auteurs déclarent qu'ils n'ont aucun conflit d'intérêts.

\section{CONTRIBUTIONS DES AUTEURS}

IFL a fait les enquêtes de terrain et rédigé le manuscrit ; OAFL a lu le manuscrit ; OPA a identifié les plantes et lu le manuscrit.

\section{REMERCIEMENTS}

Les auteurs remercient tous les tradipraticiens, commerçantes de plantes médicinales et autres personnes enquêtés pour toutes les informations qu'ils ont fournies.

\section{REFERENCES}

Adomou A. 2005. Vegetation patterns and environmental gradients in Benin. Implications for biogeography and conservation. $\mathrm{PhD}$ Thesis, Wageningen University, Wageningen, p. 136.

Adomou AC, Agbani OP, Sinsin B. 2011. Plantes. In Protection de la Nature en Afrique de l'Ouest: Une Liste Rouge pour le Bénin. Nature Conservation in West Africa: Red List for Benin, Neuenschwander P, Sinsin B, Goergen $G$ (eds). International Institute of Tropical Agriculture: Ibadan, Nigeria; 21-46.

Adomou AC, Yédomonhan H, Djossa B, Legba SI, Oumorou M, Akoègninou A. 2012. Etude ethnobotanique des plantes médicinales vendues dans le marché d'Abomey Calavi au Bénin. Int. J. Biol. Chem. Sci., 6(2): 745-772. DOI : http://dx.doi.org/10.4314/ijbcs. v6i2.18

Anonyme. 2002. Stratégie Nationale et Plan d'Action pour la Conservation de la Diversité Biologique au Bénin. Ministère de l'Environnement de l'Habitat et de l'Urbanisme (MEHU) : Cotonou.

Arbonnier M. 2009. Arbres, Arbustes et Lianes des Zones Sèches d'Afrique de l'Ouest. CIRAD-MNHN : Paris.
Assogbadjo AE, Glèlè Kakaï R, Adjallala FH, Azihou AF, Vodouhê GF, Kyndt T, Codjia JCT. 2011. Ethnic differences in use value and use patterns of the threatened multipurpose scrambling shrub (Caesalpinia bonduc L.) in Benin. Journal of Medecinal Plants Research, 5 (9): 1549-1557.

Betti JL. 2001. Vulnérabilité des plantes utilisées comme antipaludiques dans l'arrondissement de Mintom au Sud de la réserve de biosphère du Dja (Cameroun). Systematics and Geography of Plants, 71(2): 661-678. DOI: http://dx.doi.org/10.2307/ 3668709

Cunningham AB. 2001. Applied ethnobotany: people, wild plant use and conservation. People and plants, Conservation Manuel. Earthscan.

Dassou HG, Ogni CA, Yédomonhan $\mathrm{H}$, Adomou AC, Tossou M, Dougnon JT, Akoègninou A. 2014. Diversité, usages vétérinaires et vulnérabilité des plantes médicinales au Nord-Bénin. Int. J. Biol. Chem. Sci., 8(1): 189-210. DOI : http://dx.doi.org/10.4314/ijbcs.v8i1.18

Delvaux C, Sinsin B. 2003. Gestion intégrée des plantes médicinales dans la région des Monts Kouffé. In Aménagement Intégré des Forêts Naturelles des Zones Tropicales Sèches en Afrique de l'Ouest: Actes du Ilème Séminaire International sur l'Aménagement des Forêts Naturelles des Zones Tropicales Sèches en Afrique de l'Ouest, Sokpon N, Sinsin B, Eyog-Matig O (eds). Faculté des Sciences Agronomiques/Université d'AbomeyCalavi (Bénin) : Abomey-Calavi; 389405.

Dounias E, Rodrigue W, Petit C. 2000. Revue de la littérature ethnobotanique pour l'Afrique Centrale et l'Afrique de l'Ouest. Bulletin du Réseau Africain d'Ethnobotanique, 2: 5-117. DOI: http://www.documentation.ird.fr/hor/fd i:010023752 
Dzerefos CM, Witkowski ETF. 2001. Density and potential utilization of medicinal grassland plants from Abe Bailey Nature Reserve, South Africa. Biodiversity and Conservation, 10: 1875-1896. DOI : http://dx.doi.org/ 10.1023/a:1013177628331

Edward LW, Kabir MDE. 2009. Home gardening for tropical biodiversity conservation. Conserv. Biol., 23(6): 1641-1644. DOI: http://dx.doi.org/ 10.1111/j.1523-1739.2009.01267.x

Fabricant D, Farnsworth N. 2001. The value of plants used in traditional medicine for drug discovery. Environmental Health Perspectives, 109: 69-75. DOI: http://dx.doi.org/10.2307/3434847.

Fah L, Klotoé JR, Dougnon V, Koudokpon H,Fanou VBA, Dandjesso C, Loko F. 2013. Étude ethnobotanique des plantes utilisées dans le traitement du diabète chez les femmes enceintes à Cotonou et Abomey-Calavi (Bénin). Journal of Animal \& Plant Sciences, 18(1): $2647-$ 2658.

Fennell, C, Lindsey K, Mcgaw L, Sparg S, Stafford G, Elgorashi E, Grace O, Van Staden J. 2004. Assessing African medicinal plants for efficacy and safety: pharmacological screening and toxicology. Journal of Ethnopharmacology, 94: 205-217. DOI: $\quad$ http://dx.doi.org/10.1016/ j.jep.2004.05.012

Galluzzi G, Eyzaguirre P, Negri V. 2010. Home gardens: neglected hotspots of agro biodiversity and cultural diversity. Biodivers. Conserv., 19: 3635-3654. DOI: http://dx.doi.org/10.1007/s10531010-9919-5

Geldenhuys CJ, Delvaux C. 2002. Study of the distribution of Forest tree regeneration in adjacent plantation stand, Nzimankulu forest. Unpublished report, CP Wild Project: Nzimankulu.

Idohou AFR, Fandohan B, Salako VK, Kassa $\mathrm{B}$, Gbèdomon $\mathrm{R} \mathrm{C}$, Yédomonhan $\mathrm{H}$, Glèlè Kakaï RL \& Assogbadjo AE. 2014. Biodiversity conservation in home gardens: traditional knowledge, use patterns and implications for management, International Journal of Biodiversity Science, Ecosystem Services \& Management, 10(2): 89100. DOI: http://dx.doi.org/10.1080/ 21513732.2014.910554

Keirungi J, Fabricius C. 2005. Selecting medicinal plants for cultivation at Nqabara on the Eastern Cape Wild Coast, South Africa. South African Journal of Sciences, 101: 497-501.

Lalèyè FOA, Mensah S, Assogbadjo AE and Ahissou H. 2015. Diversity, Knowledge and Use of Plants in Traditional Treatment of Diabetes in the Republic of Benin. Ethnobotany Research \& Applications, 14: 231-257. DOI: http://dx.doi.org/10.17348/ era.14.0.231-257

Lawin IF, Lalèyè FOA, Agbani OP, Assogbadjo AE. 2015. Ethnobotanical assessment of the plant species used in the treatment of diabetes in the SudanoGuinean zone of Benin. Journal of Animal \& Plant Sciences, 26(3) : 41084123.

Lejoly J. 2001. La banque de données sur les plantes médicinales africaines; l'exemple de Pharmel (communication). In Réseau «Espèces ligneuses médicinale »: Compte Rendu de la Première Réunion du Réseau, Eyog-Matig O, Adjanohoun E, de Souza S, Sinsin B (eds). Institut International des Ressources Phytogénétiques: Cotonou; 91-95.

Makumbelo E, Lukoki L, Paulus JJSJ, Luyindula N. 2008. Stratégie de valorisation des espèces ressources des produits non ligneux de la savane des environs de Kinshasa: II. Enquête ethnobotanique (aspects médicinaux). Tropicultura, 26(3): 129-134.

Okou F. 2009.Vulnérabilité des ressources forestières médicinales du noyau central de la forêt classée de la Lama face aux prélèvements ethnobotaniques et estimation de leur valeur 
économique. Thèse pour l'obtention du Diplôme d'Ingénieur Agronome, Université d'Abomey-Calavi (Bénin), Abomey-Calavi, p. 135.

Salako VK, Fandohan B, Kassa B, Assogbadjo AE, Idohou AFR, Gbedomon RC, Chakeredza S, Dulllo ME, Glèlè Kakai R. 2014. Home gardens: an assessment of their biodiversity and potential contribution to conservation of threatened species and crop wild relatives in Benin. Genet Resour Crop Evol., 61: 313-330. DOI: http://dx.doi.org/10.1007/s10722-0130035-8

Sigdel SR and Rokaya MB. 2011. Utilization of plant resources in Dang district, West Nepal. Banko janakari, 21(2): 4554. DOI: http://dx.doi.org/10.3126 /banko.v21i2.9143

Sinsin B, Owolabi L. 2001. Rapport sur la Monographie de la Diversité Biologique du Bénin. MEHU: Cotonou.

Souto T, Ticktin T. 2012. Understanding interrelation ships among predictors (age, gender, and origin) of local ecological knowledge. Econ. Bot., 66:149-164. DOI: http://dx.doi.org/ 10.1007/s12231-012-9194-3

Taylor J, Rabe T, Mcgaw L, Jager A, Van Staden J. 2001. Towards the scientific validation of traditional medicinal plants. Plant Growth Regulation, 34: 23-37. DOI : http://dx.doi.org/10.1023/ a:1013310809275
Tchigankong ND. 2009. Les plantes médicinales et la problématique de la santé en Afrique: quelle orientation pour la coopération de l'Union Européenne en Afrique ? Cas du sida et de la malaria. Diplôme des hautes études européennes et internationales (DHEEI), Université de Nice, Nice, p. 130.

Traoré L, Ouédraogo I, Ouédraogo A, Thiombiano A. 2011. Perceptions, usages, et vulnérabilité des ressources végétales ligneuses dans le Sud-Ouest du Burkina-Faso. Int. J. Biol. Chem. Sci., 5(1): 258-278. DOI : http://dx.doi.org/10.4314/ijbcs.v5i1.681 03

Wagner A, Kriechbaum M, Koch MA. 2008. Applied Vulnerability Assessment of Useful Plants: A case study of Tibetan Medicinal Plants from Nepal. Bot. jahrb. Syst., 127(29): 359-387. DOI: http://dx.doi.org/10.1127/00068152/2008/0127-0359

Yapi AB, Kassi NJ, Fofie NBY Zirihi GN. 2015. Etude ethnobotanique des Asteraceae médicinales vendues sur les marchés du district autonome d'Abidjan (Côte d'Ivoire). Int. J. Biol. Chem. Sci., 9(6): 2633-2647. DOI : http://dx.doi.org/10.4314/ijbcs.v9i6.10

Zassada J. 2003. Non-timber forest products and implications for forest managers. University of Minesota, Extension service: Minesota. 